\title{
Difference between focal mechanisms of Dayao earthquake doublet sequence*
}

\author{
Xiaoshan Wang ${ }^{1}$ Xiangdong Feng ${ }^{1}$ Guiling Diao ${ }^{1, \star}$ \\ Libing Wang ${ }^{2}$ and Lingling Cai ${ }^{1}$ \\ ${ }^{1}$ Earthquake Administration of Hebei Province, Shijiazhuang 050021, China \\ ${ }^{2}$ Hongshan Benchmark Seismic Station, Longyao 055350, China
}

\begin{abstract}
Using the maximum amplitude ratios of vertical component of $\mathrm{P}$ and $\mathrm{S}$ waves recorded by a regional network, 921 focal mechanisms of Dayao earthquake doublet sequence are determined by means of synthetic seismograms of a point source of dislocation in a plane layered medium. Among them, 389 focal mechanisms are in the aftershock sequence of $M 6.2$ earthquake occurred on 21 July, 2003 and the other 532 focal mechanisms are in the aftershock sequence of M6.1 earthquake occurred on 16 October, 2003 in Dayao, Yunnan. The focal mechanism consistent parameter $a$ of the two aftershock sequences are calculated and analyzed. According to the focal mechanism consistent parameter $a$, the focal mechanisms of the first aftershock sequence are more consistent than those of the second. According to the comparison of CMT solutions of the two M6 earthquakes, the physical mechanism of the doublet in the intra-plate earthquake is very complex, and many processes are involved and interacted with each other. This doublet provides insights into earthquake clustering, triggering and stress cycling.
\end{abstract}

Key words: focal mechanism; consistent parameter $a$; Dayao earthquake doublet; difference CLC number: $\mathrm{P} 315.3^{+} 3$ Document code: A

\section{Introduction}

Earthquake is a natural phenomenon of a sudden release of energy in the Earth's crust that propagates seismic waves. Focal mechanisms of earthquakes are the basic data for studying the regional stress field. Therefore, the temporal evolution of stress field in the source region derived from the focal mechanisms of earthquakes occurred in this region is important for understanding and forecasting the process of earthquake preparation. Many seismologists have made great efforts to searching correct methods to describe the stress distribution in the processes of earthquake preparation, occurrence and development in order to understand the evolution process of the source region (Mc Kenzie, 1969; Sadovsky, 1972; Kagan, 1997; Reasenberg, 1999; Tanimoto and Okamoto, 2000; Kubo and Fukuyama, 2003). On the basis of the study on the foreshocks of Haicheng earthquake, Chen (1978) proposed that the

\footnotetext{
* Received 10 July 2009; accepted in revised form 15 September 2009; published 10 December 2009.

- Corresponding author.e-mail: dg1@eq-he.ac.cn
}

consistent parameter of focal mechanism can be used as a new parameter to judge foreshock. Diao et al (2004) developed this method and used it to forecast strong aftershocks, for example, the 1991 Datong earthquake (M5.8), the 1999 Datong earthquake (M5.6) and the 1999 Zhangbei earthquake (M5.6) (Zhao et al, 2004). They obtained the result that the focal mechanism consistency was low before strong earthquakes occurred in Jiashi in China (Diao et al, 2005), Kurile Islands area in Japan (Wang and Diao, 2005) and so on.

A large earthquake is soon followed by an event of comparable size, either on an adjacent portion of the fault that ruprured initially or a separate fault, and such event are termed doublet (Lay and Kanamori, 1980; Xu and Schwartz, 1993). The Dayao doublet occurred successively on 21 July and 16 October, 2003 in Dayao, Yunnan province induced human casualty and economic losses to a certain extent. Following the two earthquakes, a set of digital mobile seismographs was installed at the earthquake site as a near-source observation network, which could record digital data of seismic waves near the source and monitor the spatial and temporal varia- 
tion of seismic sequence in the real time. They have provided a convenient condition for studying the changes of stress field in the area of Dayao earthquake sequences. In our study, we selected the waveforms of earthquake events recorded by four or more stations from the reports compiled by Qin et al (2005) and computed the focal mechanisms of earthquakes by using the maximum amplitude ratios of vertical component of $P$ to S waves (Liang et al, 1984). On the basis of these focal mechanisms, their differences in the earthquake doublet sequence were discussed in detail by using the parameter of focal mechanism consistency, in order to provide insights into earthquake clustering, triggering and stress cycling (Lay and Kanamori, 1980; Xu and Schwartz, 1993; Kagan and Jachson, 1999).

\section{Data}

A network of seven recording stations was set up around the epicentral location of Dayao earthquake occurred on 21 July, 2003 by the Earthquake Administra- tion of Yunnan province. The distribution of stations is shown in Figure 1. The seismic geophone in Santai station was FBS-3A and those in the other stations were JC-V100-3D. The recorders in Tanhuaxiang station was EDAS-24L3 and those in the other stations were EDAS-3M with the same sampling rate of $100 \mathrm{~Hz} .2173$ aftershocks were recorded by this seismic network from 08:56 (Beijing Time) July 22 to 13:40 August 21 and the maximum aftershock was $M_{\mathrm{L}} 4.7$.

After the M6.1 earthquake occurred on 16 October, 2003, a temporary seismic network composed of five digital stations was deployed around the main shock by the Earthquake Administration of Yunnan province (Figure 1). The seismic geophones in all stations were JC-V100-3D and the recorders in all stations were EDAS-3M with the same sampling rate of $100 \mathrm{~Hz} .3793$ aftershocks were recorded from 05:13 (Beijing Time) October 17 to $15: 00$ November 17 and the maximum aftershock was $M_{\mathrm{L}} 4.6$.

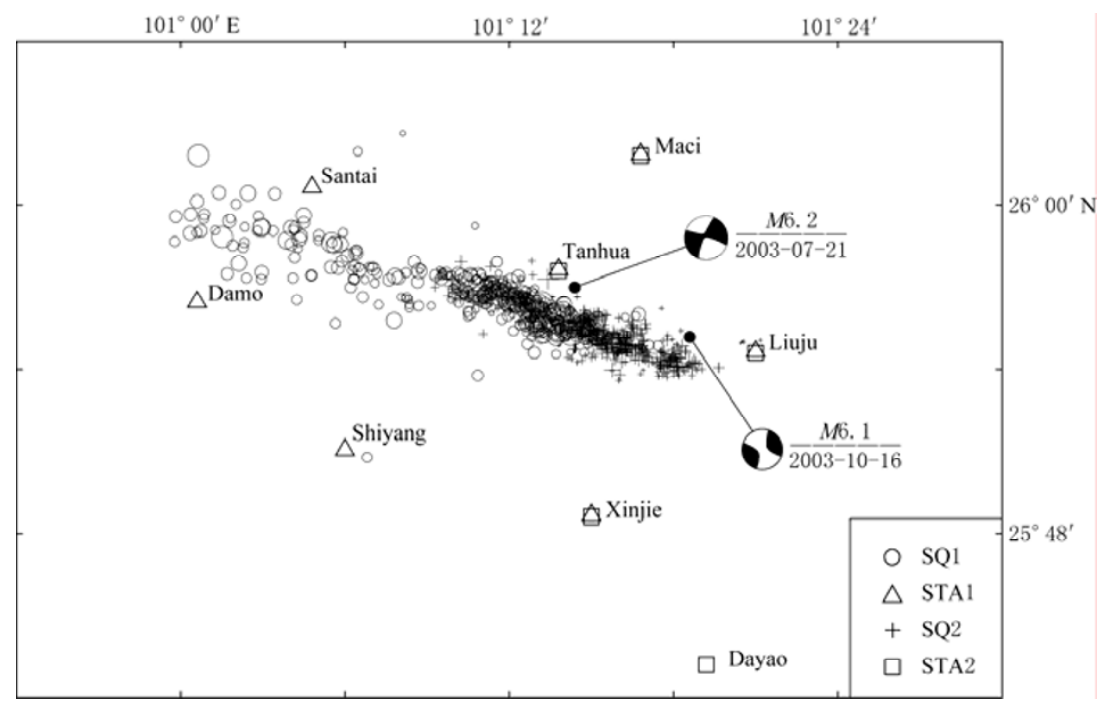

Figure 1 Epicentral distribution of Dayao earthquake doublet sequence with focal mechanisms and distribution of station. The circles indicate the epicenters of aftershocks of M6.2 earthquake with focal mechanisms and the triangles denote the stations deployed after the $M 6.2$ earthquake; the crosses represent the epicenters of M6.1 earthquake with focal mechanisms and the squares are the stations deployed after the $M 6.1$ earthquake.

The spatial distribution of epicenters with focal mechanism in the two M6 Dayao earthquake sequences shows that the two M6 earthquakes extended from NW to SE, but the two aftershock sequences extended from SE to NW, which dovetailed each other (Figure 1). The spread of the first M6.2 earthquake is dispersed and longer than that of the second, while the distribution of the second M6.1 aftershock sequence is more centralized. The focal depth has a significant change with time from the 30-point running average. The focal depth of the first M6.2 earthquake underwent a deep-shallowmedium deep-shallow evolutionary process; the second earthquake sequence occurred mostly in the shallow crust (Figure 2). 


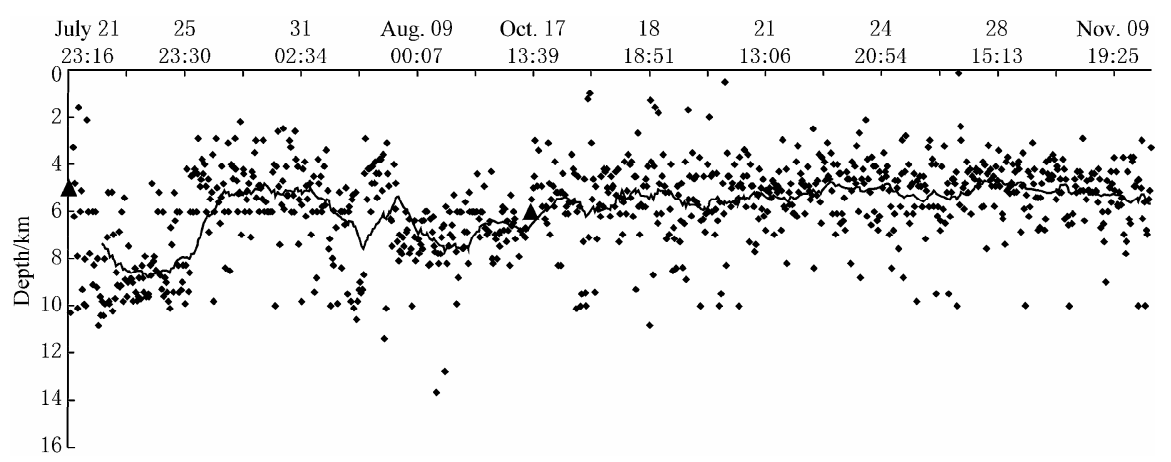

Figure 2 Focal depth change with seismic sequence. The triangle indicates the occurrence time of $M 6.2$ and M6.1 earthquake. The black line is the 30-point running average of the depth.

\section{Calculation and analysis of focal mechanism}

The focal mechanisms of the two M6 earthquake sequences are determined by means of synthetic seismograms of a point source of dislocation in a plane layered medium, using the maximum amplitude ratios of vertical component of $\mathrm{P}$ and $\mathrm{S}$ waves recorded by a regional network (Liang et al, 1984). We select strictly some high-quality data to determine the focal mechanism. The selecting rules are as follows: (1) the event should be recorded by at least four stations; (2) the waveform has at least a clear first motion. The parameters of Chuxiong (west) velocity model (Hu et al, 1986) are used. We calculate 389 focal mechanisms in the aftershock sequence of M6.2 earthquake occurred on 21 July, 2003 and determine 532 focal mechanisms in the aftershock sequence of M6.1 earthquake occurred on 16 October, 2003.

Figure 3 shows the normalized frequency distribution per $10^{\circ}$ for different parameters of the focal mechanisms of the two M6 Dayao earthquake sequences. In the statistics of nodal-plane parameters, the fault plane and auxiliary plane are not separated from each other, but are calculated together. On the basis of the statistics of different parameters, we have found that the focal mechanism parameters of the first M6.2 sequence are more consistent than those of the second, which shows that the focal area is still controlled by the focal stress field and in a high stress level before the M6.1 earthquake. The statistics result of the M6.1 sequence is dispersed and the stress regime is transformed to a low level after the $M 6.1$ earthquake.

The directions of stress field are expressed by three principal stress axes of $\sigma_{1}, \sigma_{2}$ and $\sigma_{3}$, which are perpen- dicular to each other; and the orientations of focal mechanism are shown by three stress axes of $P, N, T$ that are also perpendicular to each other (Figure 4). Then, the consistent parameter for defining focal mechanism and tectonic stress field is (Wang and Diao, 2005)

$$
a=\alpha+\beta+\gamma
$$

where $\alpha=\angle \sigma_{1} O P, \beta=\angle \sigma_{2} O N, \gamma=\angle \sigma_{3} O T$. The maximum value for the consistent parameter is $270^{\circ}$ and the minimum value is $0^{\circ}$.

Scholz (1990) presented a criterion to simply distinguish large and small earthquakes under the condition that only physical cause of rupture was considered. The criterion is a relation between total rupture scale and thickness of the rupture loop. Large earthquake refers to the event with the total rupture scale larger than or equal to the thickness of the rupture loop. When earthquake is large, the rupture scale has to extend horizontally and its mean aspect ratio increases gradually. As a result, its upper end extends to the ground and its lower end to the bottom of the rupture loop. Certainly, the thickness of rupture loops differs in locations and thus the magnitude for division is also different. For example, the rupture loop of San Andres fault is only about $15 \mathrm{~km}$ thick and its magnitude for division is 6-6.5; while the rupture loop in the underthrust zone is much thicker, therefore its magnitude for division is about 7.3. Whether an earthquake is large or small depends on different scale relations, which shows different geometrical conditions and boundaries. When we consider quantitatively the role of earthquakes in geological structure, only large earthquakes are included. Therefore, we take $P, N, T$ axes of the M6.2 earthquake as a reference frame of stress. The azimuths and dips of principal stress axes $\sigma_{1}$, 
$\sigma_{2}$ and $\sigma_{3}$ are $155^{\circ}$ and $11^{\circ}, 321^{\circ}$ and $78^{\circ}, 65^{\circ}$ and $3^{\circ}$, respectively. Then the consistent parameter $a$ of each focal mechanisms and tectonic stress field is calculated in the 3-D space.
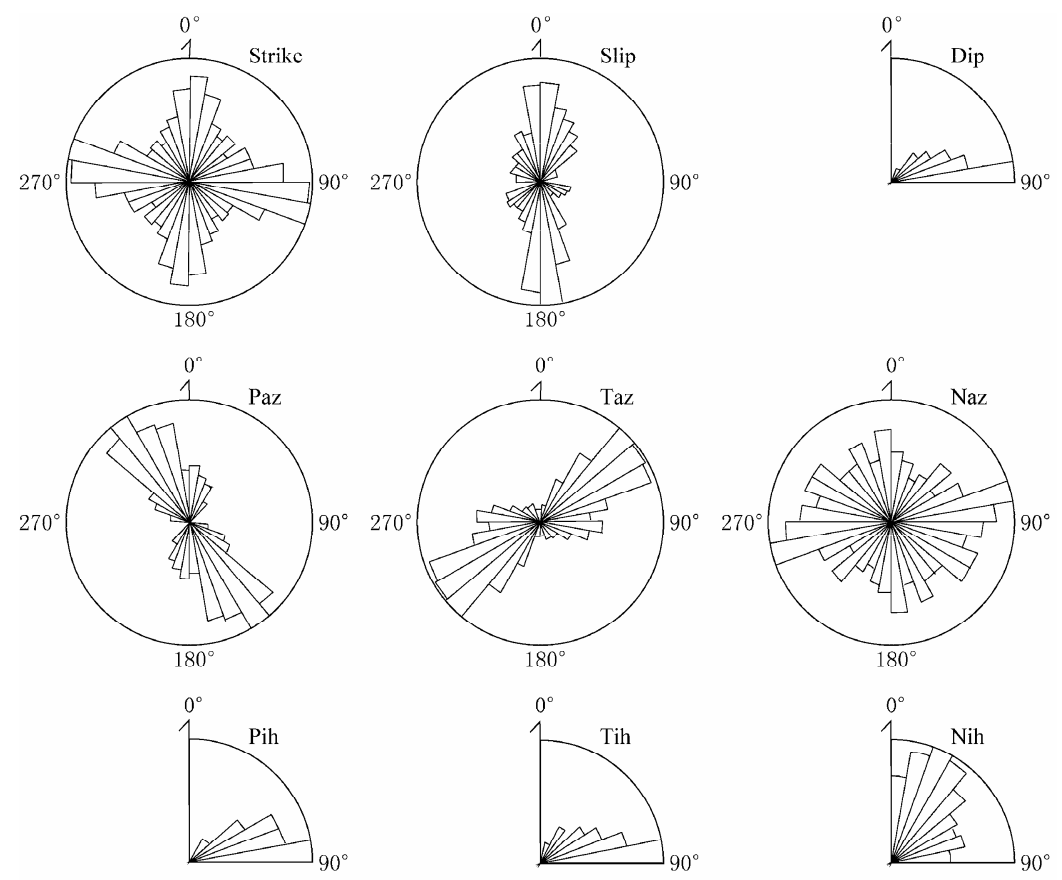

(a)
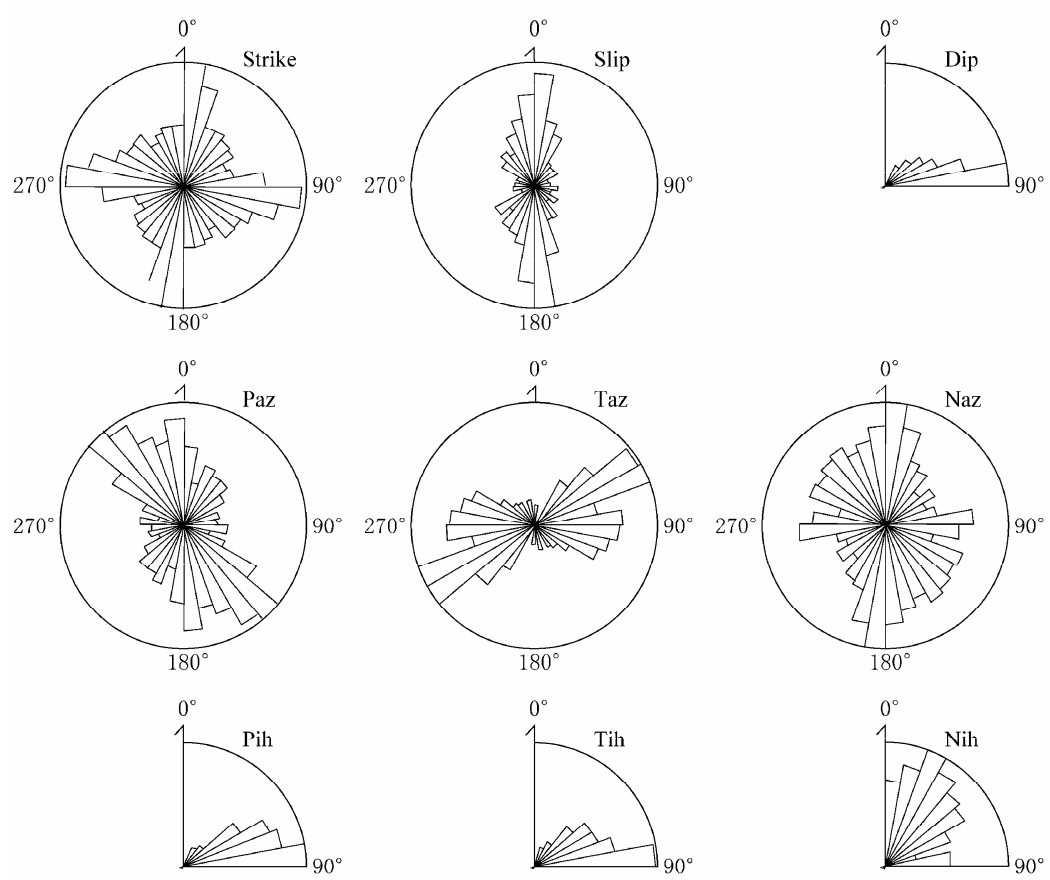

(b)

Figure 3 Normalized frequency distribution per $10^{\circ}$ for different parameters of focal mechanisms. (a) M6.2 earthquake sequence; (b) M6.1 earthquake sequence.

The consistent parameter $a$ calculated for each sequence focal mechanisms of Dayao doublets is shown in Figure 5. The average value and standard deviation of the consistent parameter $a$ of the M6.2 earthquake se- quence are $161.78^{\circ}$ and $38.88^{\circ}$, respectively (Figure 5a); while the average value and standard deviation of the consistent parameter $a$ of the M6.1 earthquake sequence is $175.89^{\circ}$ and $37.72^{\circ}$, respectively (Figure $5 b$ ). There is 
a significant difference in the stress regime between the two seismic sequences. The consistent parameter $a$ of the former sequence is smaller than that of the latter, which indicates that the aftershocks of the M6.2 earthquake are controlled by the main shock and the stress state of the focal area is at a high level. After the M6.1 earthquake occurred on 16 October, the release of energy and the stress field of the focal area have a process of adjustment. Many factors may be involved in this complex adjustment process, such as rupture heterogeneity of the M6.2 earthquake, stress triggering of the M6.2 earthquake (Hua et al, 2006), fluid invasion into fractures, the regional stress field, etc.

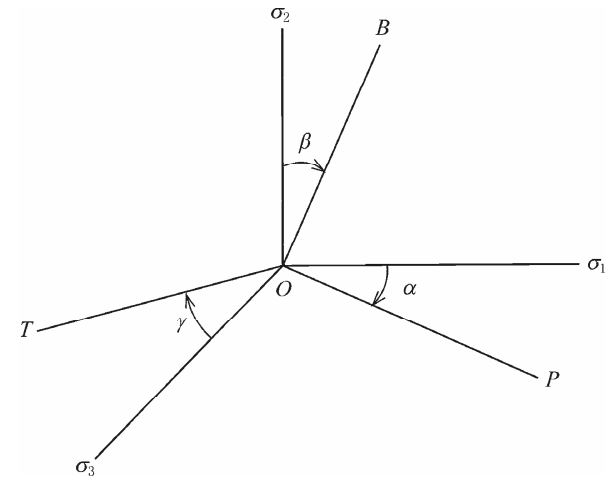

Figure 4 Included angles for three principal axes $N, P$ and $T$ of tectonic stress field and principal stress axes of focal mechanism.
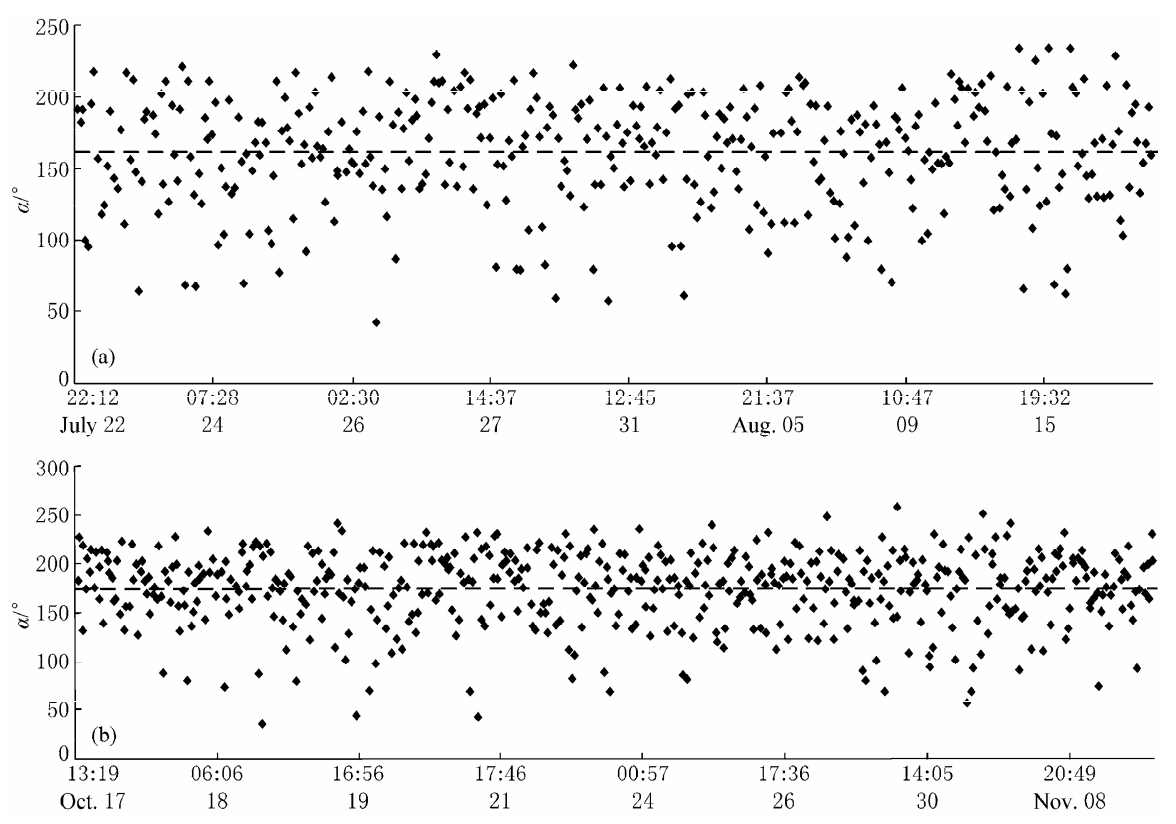

Figure 5 Consistent parameter $a$ calculated for each sequence focal mechanisms of the Dayao doublets. The dashed line indicates the average of consistent parameters. (a) indicates the consistent parameters of the $M 6.2$ earthquake; (b) indicates the consistent parameters of the $M 6.1$ earthquake.

We put two aftershock sequences together to calculate the consistent parameter $a$ (26-point moving average) for each focal mechanism with the magnitude of $M_{\mathrm{L}} \geq 2.5$ (Figure 6). We found that there is an interesting phenomenon that consistent parameter $a$ decreased to a very low level in the later period of the M6.2 earthquake though the number of the solution is very small because the temporary seismic network stopped recording at 13:40 on August 21, 2003.

From Figure 1 and Table 1, we have found that the two solutions are basically identical and only the non-shear component of the latter is larger than that of the former (Figure 1). The DC (Double Couple) component of the M6.2 earthquake is $95 \%$ and the $M 6.1$ is $75 \%$. The physical causes of this phenomenon are not yet well understood. It is likely that more than one process is involved, such as the geometrical fault complexity, tensile failure, dilatancy, and fluid flow in faulting (Julian, 1998). The generation of compound earthquakes indicates heterogeneity in the faulting process, which has been attributed to a specific pattern of fault plane heterogeneity consisting of closely spaced asperities (areas with increased strength) on the fault contact plane such that the failure of one asperity trig- 
gers immediate slip of adjacent asperities (Horikawa, 2001). Wang et al (2007) inferred that there are likely two faults intersecting with each other in the Dayao focal area based on the 3-D $v_{\mathrm{P}}, v_{\mathrm{S}}, Q_{\mathrm{P}}$ structures obtained by inversion of travel time and waveform data. The 3-D imaging results show an obvious WNW faults with low
$v_{\mathrm{P}}$ and low $Q_{\mathrm{P}}$ anomalies and a ENE fault that dips westward and exhibits low $v_{\mathrm{P}}$, low $v_{\mathrm{S}}$ and low $Q_{\mathrm{P}}$ features. The ENE fault limited the seismicity to extend eastwards and there may be asperity in the south of the fault.

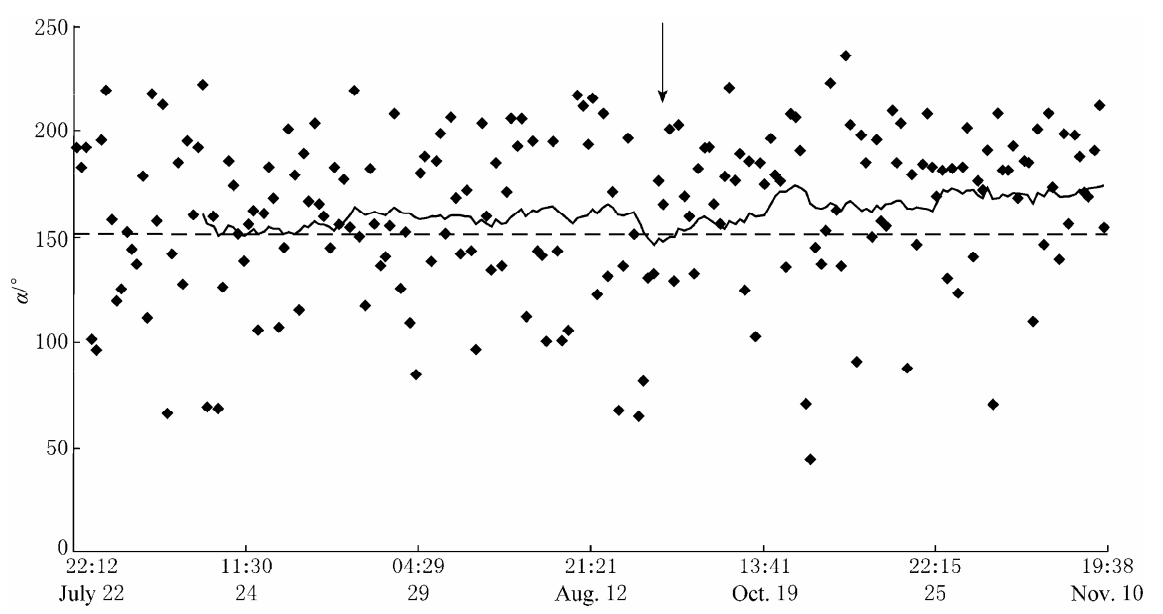

Figure 6 Consistent parameter $a$ (26-point moving average) calculated for each focal mechanism with magnitude $M_{\mathrm{L}} \geq 2.5$. The dashed line indicates the threshold value of consistent parameter; the arrow indicates the occurrence time of the M6.1 earthquake.

Table 1 CMT solutions of two $M 6$ strong earthquakes

\begin{tabular}{|c|c|c|c|c|c|c|c|c|c|c|c|c|c|}
\hline \multirow{2}{*}{ No. } & \multicolumn{2}{|c|}{$P$ axis } & \multicolumn{2}{|c|}{$T$ axis } & \multicolumn{2}{|c|}{$N$ axis } & \multicolumn{3}{|c|}{ Nodal plane 1} & \multicolumn{3}{|c|}{ Nodal plane 2} & \multirow{2}{*}{ DC } \\
\hline & $\mathrm{Pl} /^{\circ}$ & $\mathrm{Az} /{ }^{\circ}$ & $\mathrm{Pl} /^{\circ}$ & $\mathrm{Az} /^{\circ}$ & $\mathrm{Pl} /^{\circ}$ & $\mathrm{Az} /{ }^{\circ}$ & Strike $/^{\circ}$ & Dip $/^{\circ}$ & $\mathrm{Rake}^{\circ}$ & Strike $/^{\circ}$ & $\mathrm{Dip} /{ }^{\circ}$ & $\mathrm{Rake}^{\circ}$ & \\
\hline 1 & 11 & 155 & 3 & 65 & 78 & 321 & 200 & 80 & -6 & 291 & 84 & -170 & $95 \%$ \\
\hline 2 & 2 & 333 & 1 & 243 & 88 & 135 & 18 & 88 & -1 & 108 & 89 & -178 & $75 \%$ \\
\hline
\end{tabular}

Note: The data are from http://www.globalcmt.org/CMTsearch.html

\section{Discussion and conclusions}

A doublet on a local scale is a pair of seismic events with similar waveforms, but different origin times, and is considered to be the result of stress release on the same fracture (Moriya et al, 2002). Through analysis on the detailed differences of the focal mechanisms of Dayao earthquake doublet sequence, we have found that the stress regime in the focal area have a process of transform. Earthquake doublets not only present particular challenges for seismic hazard assessment after a large event, but also provide insights into earthquake clustering, triggering and stress cycling, which is a powerful tool for detailed studies of seismotectonic structures and propagation characteristics of seismic waves (Augliera et al, 1995).

The dominant orientation of each parameter of focal mechanisms approaches the CMT solutions; the strike of the nodal plane is consistent with the long axis direction of epicentral distribution area. The strikes of nodal plane are WNW-ESE, which are consistent with the extension of aftershock distribution and the dip angle is nearly vertical. The strike-slip accounts for majority of the focal mechanisms of aftershock sequences. The azimuth of $P$ axis is NNW, the azimuth of $T$ axis is ENE and the dip angle is nearly $0^{\circ}$. The dip angle of $N$ axis is nearly vertical. This characteristic is similar to the orientation of local tectonic stress field and is consistent with the focal mechanisms of medium and strong earthquakes occurred in recent years nearby (Wu et al, 2004). The analysis above shows that the orientation of regional stress field is relative stable.

According to the comparison of CMT solutions of the two $M 6$ earthquakes, it is likely that more than one process is involved, such as the geometrical fault complexity, stress trigger, fluid and fault plane heterogeneity. Recently, Felzer et al (2004) demonstrated that the sta- 
tistics of earthquake data in several catalogues were consistent with a single triggering mechanism responsible for the occurrences of aftershocks, foreshocks and multiplets; and they were caused by the same physical process. They found that the high rate of multiplets in several subduction zones might be explained simply by a high regional aftershocks rate and earthquake density. However, the physical mechanism of the doublet in the intra-plate earthquake is very complex, and many processes are involved and interacted with each other.

Acknowledgements This study is supported by the Program of the Eleventh Five-year Plan of China (2006BA-01B02-01-01). Thanks to Dr. Wessel P and Dr. Smith W H F for providing us with the software GMT. We thank Lishen Liu and Rui Yang for helping us calculating focal mechanisms.

\section{References}

Augliera P, Cattaneo M and Eva C (1995). Seismic multiplets analysis and its implication in seismotectonics. Tectonophysics 248: 219-234.

Chen Y (1978). Consistency of focal mechanism as a new parameter in describing seismic activity. Chinese J Geophys 21(2): 142-159 (in Chinese with English abstract).

Diao G L, Wang H T, Gao G Y, Long H Y and Nie X H (2005). A deflection process for stress field of the Jiashi strong earthquake sequence. Chinese $J$ Geophys 48 (5): 1062-1 068 (in Chinese with English abstract).

Diao G L, Zhao Y P, Chuo Y Q, Wang Q C, Gao J C, Cao S C, Wang Y and Zhu Z X (2004). Coherence characteristics of focal mechanism solutions of later-period strong aftershocks. Inland Earthquake 18(3): 202-206 (in Chinese with English abstract).

Felzer K R, Abercrombie R E and Ekström G (2004). A common origin of aftershocks, fore-shocks, and multiplets. Bull Seism Soc Amer 94: 88-98.

Horikawa H (2001). Earthquake doublet in Kagoshima, Japan: Rupture of asperities in a stress shadow. Bull Seism Soc Amer 91: 112-127.

Hu H X, Lu H X, Wang C Y, He Z Q, Zhu L B, Yan Q Z, Fan Y X, Zhang G Q and Deng Y E (1986). Study on explosion earthquakes of crustal structure in western Yunnan Province. Chinese J Geophys 29(2): 133-143 (in Chinese with English abstract).

Hua W, Liu J, Zheng S H and Chen Z L (2006). The characteristic analysis and seismic triggering study of the Dayao M6.2 and M6.1 earthquake sequences in 2003. Earthquake Research in China 22(1): 10-23 (in Chinese with English abstract).

Julian B R, Miller A D and Foulger G R (1998). Non-double-couple earthquakes: 1.Theory. Reviews of Geophysics 36(4): 525-549.
Kagan Y Y (1997). Seismic moment-frequency relation for shallow earthquakes: Regional comparison. J Geophys Res 102: 2 835-2 852.

Kagan Y Y and Jackson D D (1999). Worldwide doublets of large shallow earthquakes. Bull Seism Soc Amer 89: 1 147-1 155.

Kubo A and Fukuyama E (2003). Stress field along the Fyukyu Arc and the Okinawa Trough inferred from moment tensors of shallow earthquakes. Earth Planet Sci Lett 210: 305-316.

Lay T and Kanamori H (1980). Earthquake doublets in the Solomon Islands. Phys Earth Planet Inter 21: 283-304.

Liang S H, Li Y M, Shu P Y and Zhu P D (1984). On the determining of source parameters of small earthquakes by using amplitude ratios of $\bar{P}$ and $\bar{S}$ from regional network observations. Chinese J Geophys 27(3): 249-257 (in Chinese with English abstract).

Mc Kenzie D P (1969). The relationship between fault plane solutions for earthquakes and directions of principal stresses. Bull Seism Soc Amer 59: 591-601.

Moriya H, Nakazato K, Niitsuma H and Baria R (2002) Detailed fracture system of the Soultz-sous-Forêts HDR field evaluated using microseismic multiplet analysis. Pure Appl Geophys 159: 517-541.

Qin J Z, Ye J Q, Cai S P and Liu X J (2005). Reports on Observation of Dayao Earthquake in Yunnan Province Recorded in the Near Source Digital Seismic Network and the Modern New Parameters. Seismological Press, Beijing, 1-384. (in Chinese).

Reasenberg P A (1999). Foreshock occurrence before large earthquakes. $J$ Geophy Res 104: 4755-4 768.

Sadovsky M A (1972). The processes preceding strong earthquake in some regions of middle Asia. Tectonophysics 14(3/4): 295-307.

Scholz C H (1990). The Mechanics of Earthquakes and Faulting. Cambridge University Press, New York, 187-189.

Tanimoto $\mathrm{T}$ and Okamoto $\mathrm{T}$ (2000). Change of crustal potential energy by earthquakes: An indicator for extensional and compressional tectonics. Geophys Res Lett 27: 2313-2 316.

Wang J G and Diao G L (2005). Consistent CMT solutions from Harvard University before great earthquakes in Kurile Islands and its significance for earthquake prediction. Acta Seismologica Sinica 18(2): 189-195.

Wang W J, Chen L, Chen Q F and Liu J (2007). Velocity and attenuation structures in the focal area of 2003 Dayao earthquakes. Chinese J Geophys 50(3): 770-779 (in Chinese with English abstract).

Wu J P, Ming Y H and Wang C Y (2004). Source mechanism of small-moderate earthquakes and tectonic stress field in Yunnan Province. Acta Seismologica Sinica 17(5): 509-517.

Xu Z and Schwartz S Y (1993). Large earthquake doublets and fault plane heterogeneity in the northern Solomon Islands subduction zone. Pure Appl Geophys 140: 365-390.

Zhao Y P, Diao G L, Gao J C, Wang Q C, Du Y C and Zhu Z X (2004). Consistency of the focal mechanisms before some strong aftershocks in Zhangbei earthquake series. North China Earthquake Sciences 22(1): 1-4 (in Chinese with English abstract). 